\title{
Multipactor in Coaxial Line Induced by DC Bias
}

\author{
Gennady Romanov, Fermilab, USA and Chaoen Wang, National Synchrotron Radiation \\ Research Center (NSRRC), Taiwan
}

\begin{abstract}
High DC voltage applied between outer and inner electrodes of coaxial transmission lines is considered and actually is an effective remedy against multipactor in these lines. However, the high voltage bias being below certain level itself initiates multipacting. In this technical note the experimental manifestation and numerical simulations of the phenomenon are presented.
\end{abstract}

\section{Experimental observation.}

Mulitpactor (MP) in the coaxial transmission lines with DC bias voltage up to $\pm 2 \mathrm{kV}$ applied to the inner conductor was observed during conditioning of the power couplers for KEKB superconducting cavities [1,2]. Also, the phenomena of multipactor in the coaxial lines with applied high voltage bias was recently observed at NSRRC during the high-power conditioning of two power input couplers. Mechanical design of the KEK coupler which is identical to the tested ones at NSRRC is shown in Fig.1. Vacuum part of the coupler consists of two 50 Ohm coaxial parts: the shorter part $(50 \mathrm{~mm})$ that contains ceramic window and has inner radius of outer conductor $76 \mathrm{~mm}$ and outer radius of inner conductor of $33 \mathrm{~mm}$, and the longer part $(\approx 700 \mathrm{~mm})$ with inner radius of outer conductor $60 \mathrm{~mm}$ and outer radius of inner conductor of $26 \mathrm{~mm}$. It also includes the tapers and the open-ended part. The inner conductor of these couplers including the ceramic RF window was originally designed for the SRF cavities for TRISTAN accelerator. Their outer conductors were made in-house at NSRRC. The coupler design allows to apply DC bias voltage to the central conductor. The experimental set up for high power tests of the couplers at NSRRC is shown in Fig.2.

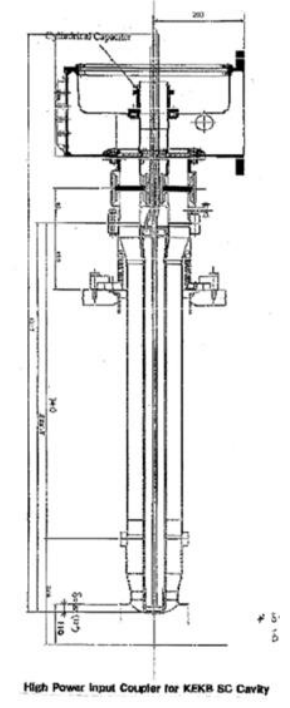

Figure 1: KEK high power input coupler for KEKB SC cavity [1]. 


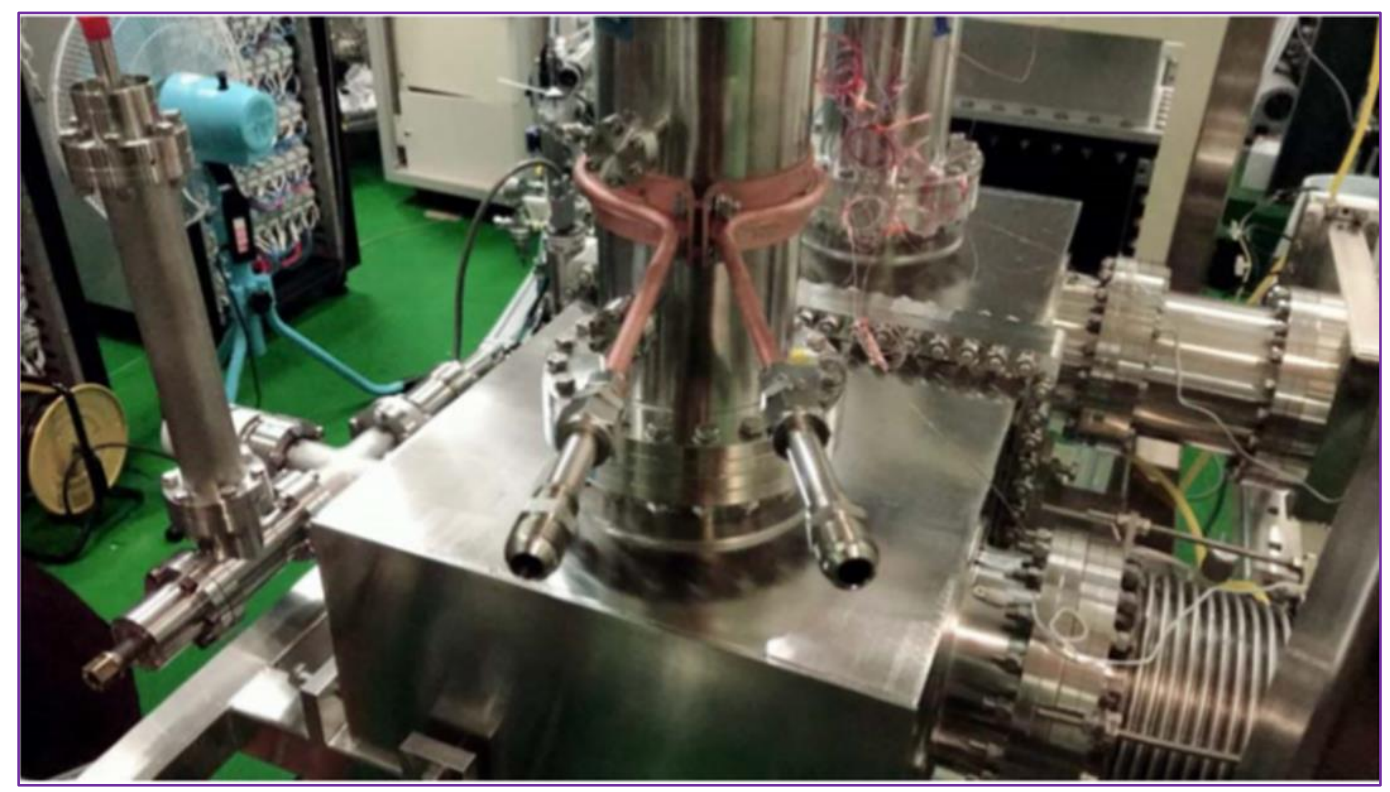

Figure 2: The experimental set up at NSRRC for high power tests of the couplers.

In the experiments at NSRRC the bias voltage was scanned from $-2 \mathrm{kV}$ to $2 \mathrm{kV}$ (the sign is related to the central conductor), operating frequency of traveling wave was $500 \mathrm{MHz}$, and the intense MP was observed at bias voltage of $\approx 1.1 \mathrm{kV}$ and RF power above $70 \mathrm{~kW}$ (see Fig.3), while the coupler after conditioning did not show any signs of MP at this power level during tests without bias. The high power test results led to the suggestion that while DC bias voltage above certain value ( $>\approx 2 \mathrm{kV}$ for both positive and negative bias ) is a powerful tool to suppress MP in coaxial lines [3], at lower voltages it can itself induce MP at RF power levels that without bias were free of multipactor.

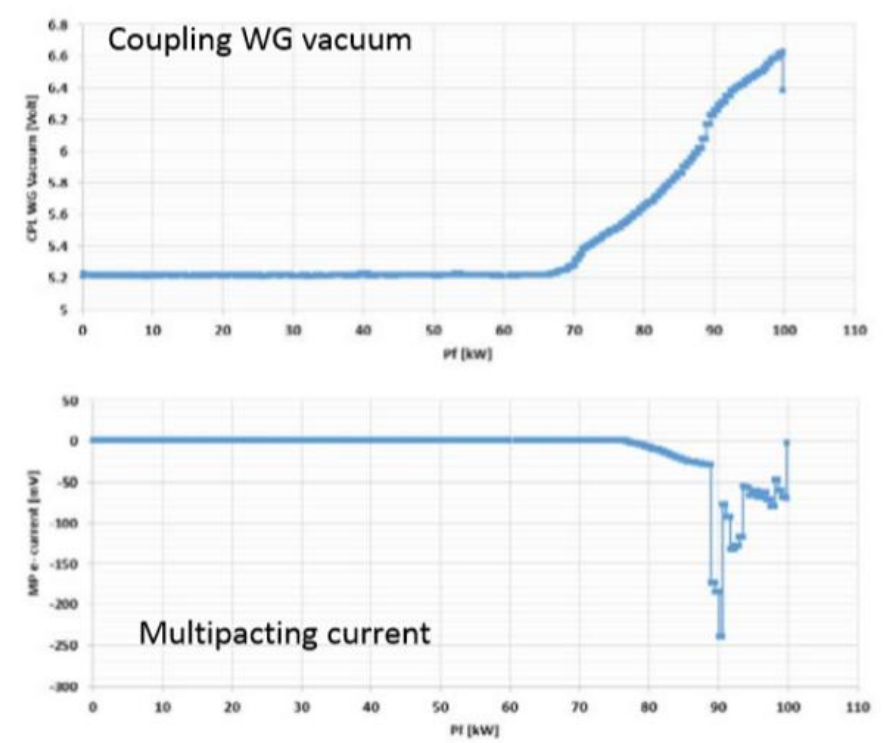

Figure 3: Indication of multipactor in the coupler at bias voltage $1100 \mathrm{~V}$ during high power test up to RF power level of $100 \mathrm{~kW}$. 
It was decided to check both regular coaxial parts by simulations with CST Particle Studio to evaluate MP development after application of high voltage bias.

\section{CST models}

The CST models of both coaxial parts of the coupler were the short pieces of coaxial waveguides of equal lengths per comparability reason $(150 \mathrm{~mm})$. The models were provided with two coaxial ports (see Fig.4), and the transient solver built in PIC solver of CST Particle Studio was used to simulate RF fields. Two ports allow to generate field of any standing wave ratio, but in present simulations only pure traveling wave was used. Mesh was rectangular since both transient solver and PIC solver require that. Number of mesh cells was $6.8 \mathrm{e} 6$, maximal size of cell was $1.3 \mathrm{~mm}$.
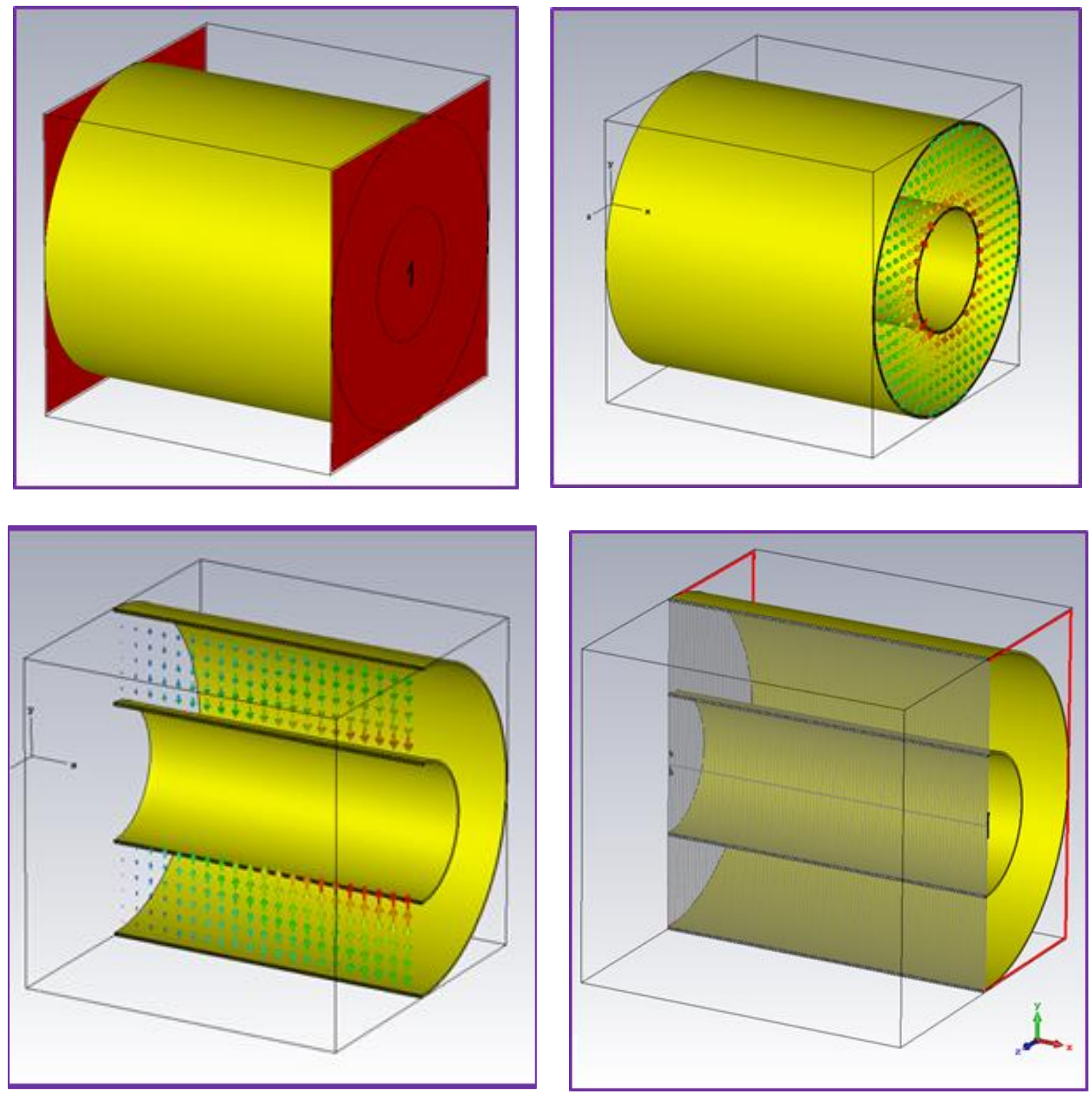

Figure 4: The $150 \mathrm{~mm}$ long coaxial model. There are shown ports, port mode, snap-shot of crosssection of traveling wave, rectangular mesh. 
To simulate high voltage bias, the electrostatic solver built in PIC was used. This solver also used the same rectangular mesh. Direction of bias field was arbitrary and could be positive (positive potential on the central conductor) or negative (see Fig.5).
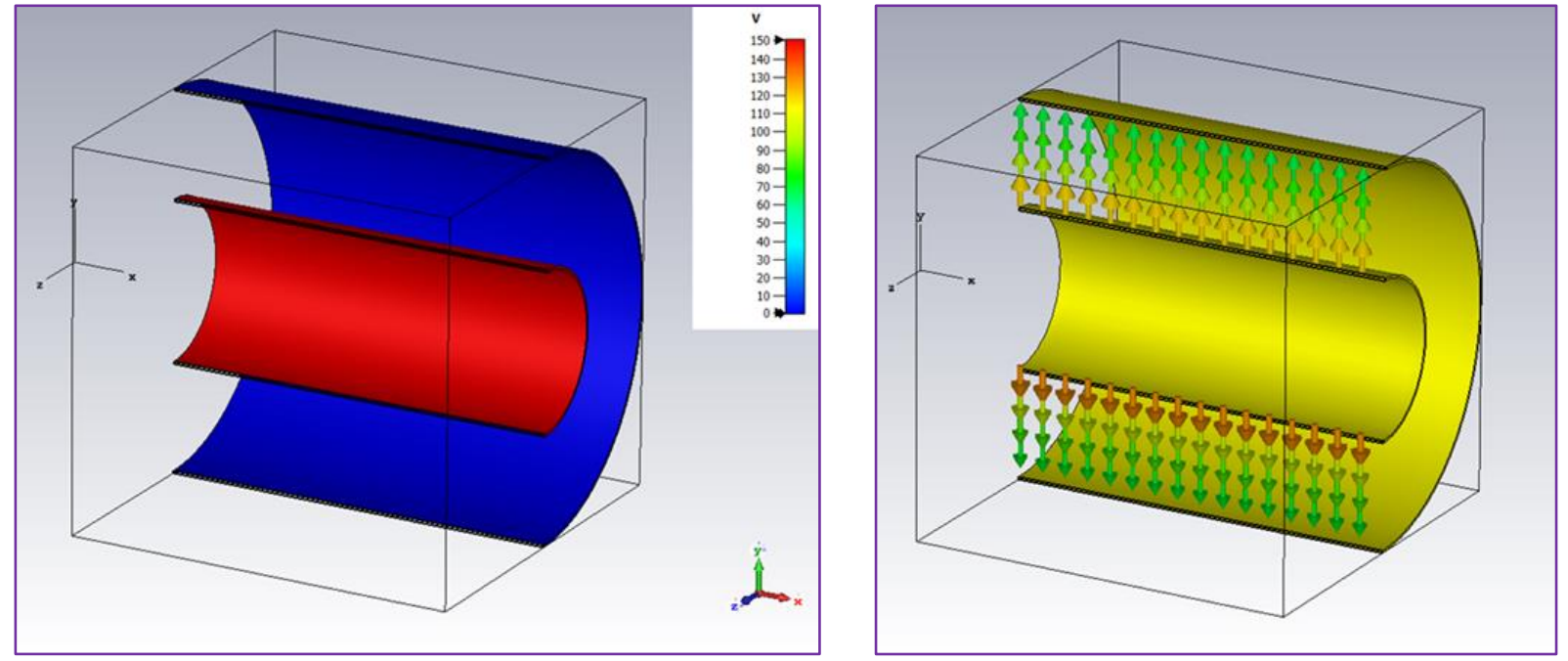

Figure 5: Potentials (positive on central conductor), and corresponding electrostatic field.

The area particle sources with gaussian emission model were placed on each electrode. The sources consisted of number of emitting points pre-defined by user (see Fig.6). The particle sources emitted rectangular $1 \mathrm{~ns}$ (half of RF period) long pulses of current in the beginning of simulation. Energies of initial electrons were uniformly distributed over 0-8 eV interval; no angle spread was assigned. Note, that these settings work for initial particles only, during simulations the parameters of secondary particles are governed by SEY model.
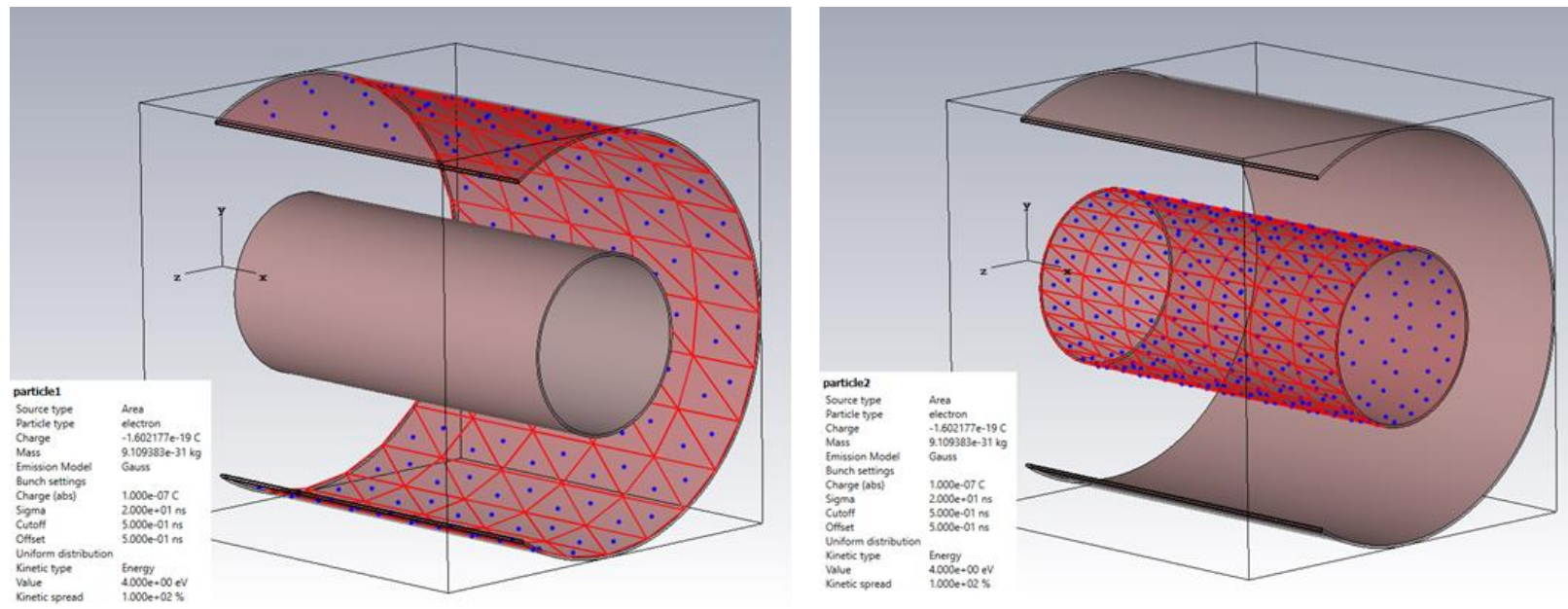

Figure 6: Area particle sources and their parameters.

The simulations were performed with space charge effects. So, typically when multiplication of particles is developing, the number of particles in the volume gradually comes to saturation (see 
Fig.7). The plateau of the number of particles is convenient to calculate the secondary space charge limited multipactor parameters, since they are essentially constant at saturation.

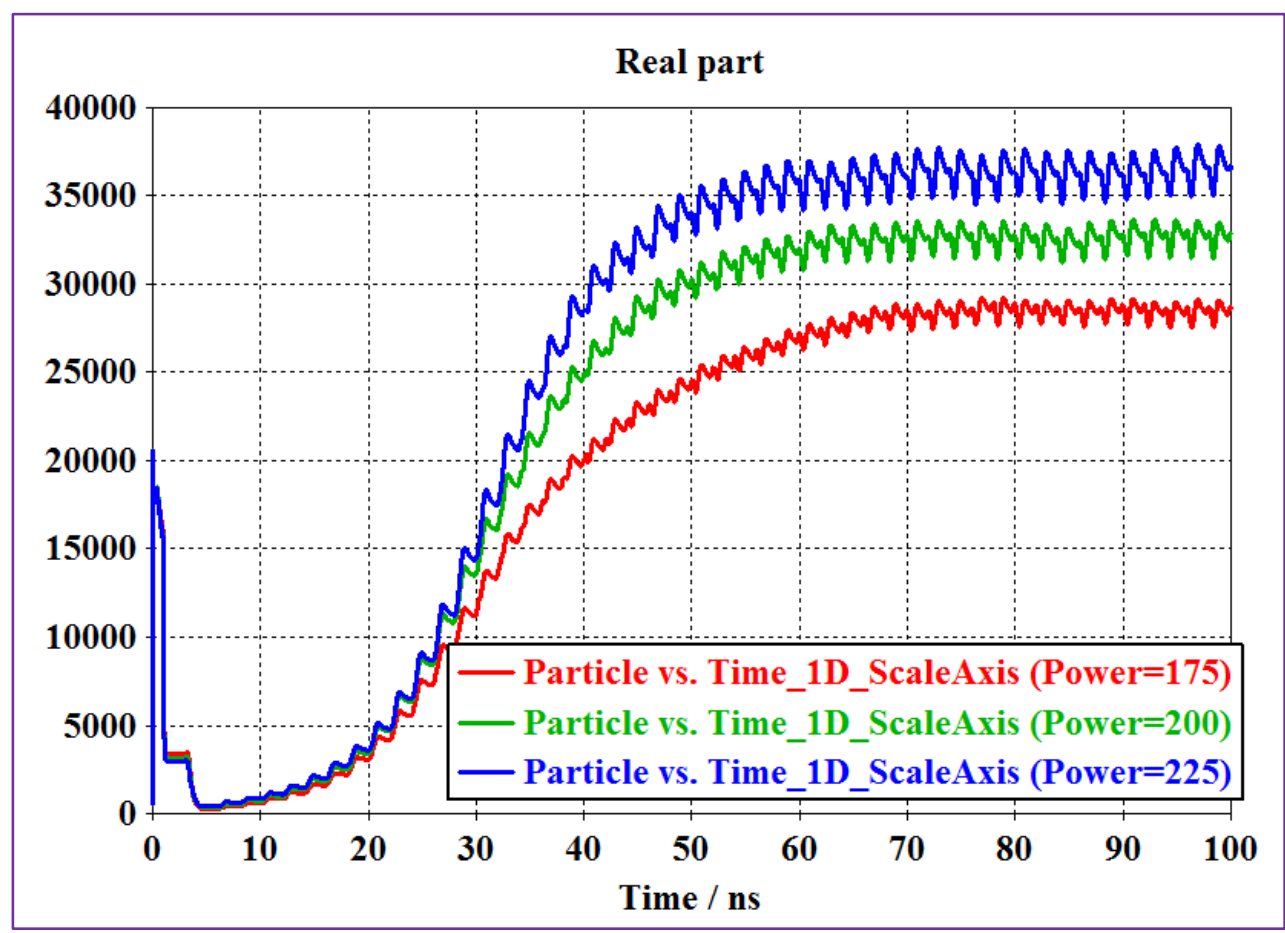

Figure 7: Example of particle number saturation in the longer coaxial part of the coupler at bias voltage of $-1.3 \mathrm{kV}$ and RF power of $225 \mathrm{~kW}$ (blue), $200 \mathrm{~kW}$ (green) and $175 \mathrm{~kW}$ (red).

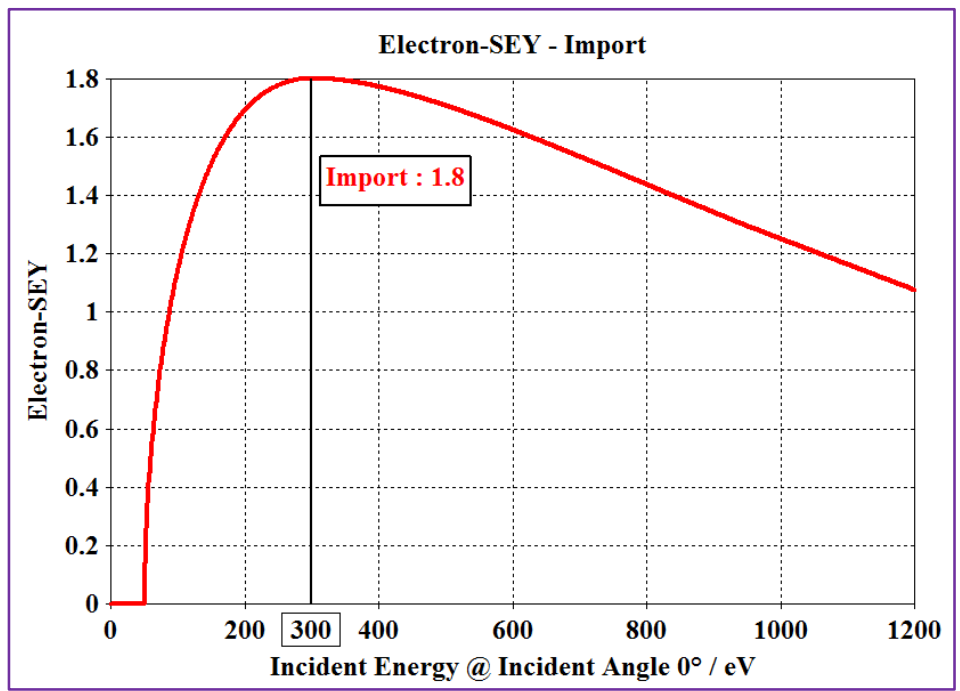

Figure 8: The SEY function of true emission used for MP simulation in the coaxial model, its maximal yield is 1.8 at $300 \mathrm{eV}$, first crossover is $\mathrm{W}_{1}=89 \mathrm{eV}$, second crossover is $\mathrm{W}_{2}=1289 \mathrm{eV}$.

\section{Multipactor in the longer part.}

The model is a piece of coaxial line with the inner radius of outer conductor $\mathrm{R}=60 \mathrm{~mm}$; the outer radius of inner conductor $\mathrm{r}=26 \mathrm{~mm}$ and the length of $150 \mathrm{~mm}$. 
The simulations with CST PIC solver in the coaxial model with bias were performed using simple imported SEY function (true emission only, shown in Fig.8). The reason for that is the use of GPU accelerator in these simulations, which currently supports only imported SEY models. Initial energy of secondary electrons had Maxwellian distribution with maximal probability at 7.5 eV (CST's default value). Also, the dependency of secondary electron yield on incident angle was considered by default in all simulations [5].

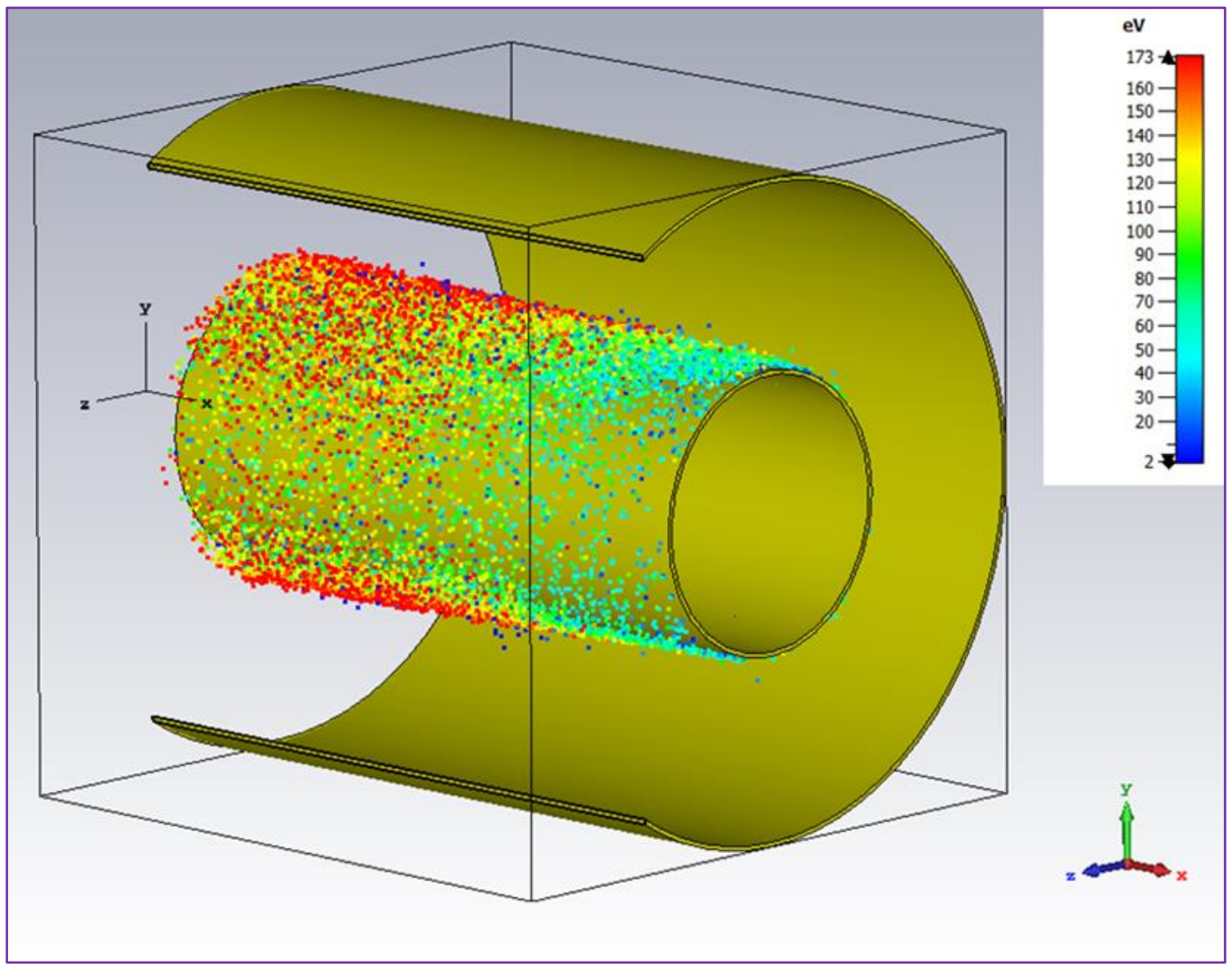

Figure 9: Snapshot of one-side multipacting on the central conductor at $\mathrm{P}=90 \mathrm{~kW}$ and positive bias of $1100 \mathrm{~V}$. Energy of particles indicated by color changes along the conductor because RF electromagnetic wave is traveling.

According to Somersalo's susceptibility chart [3,4] MP barriers for one-side MP are within interval $\approx 55-700 \mathrm{~kW}$. In our trial simulations without bias MP started at $\approx 100 \mathrm{~kW}$ and went up to $\approx 800 \mathrm{~kW}$. At the power levels of interest up to $200 \mathrm{~kW}$ it was dominantly one-side MP on the outer conductor of 6-9 orders that were merged into a single barrier. This one side multipacting in coaxial lines is determined by a radial RF field gradient that acts as a virtual returning force. Because of very high order this MP barrier is weak, and apparently because of that it was not noticed in the tests of the conditioned coupler without bias.

The simulations with bias revealed that the bias voltage acts as a real returning force and initiates one-side MP. The simulations indicated possibility of one-side MP development on both electrodes - on the outer electrode with negative bias and on the central electrode with positive bias (see also 
[3]). The one-side MP on the central electrode with positive bias (shown in Fig.9) was considered first.

The RF power level, at which this MP can develop, depends strongly on the applied bias voltage. To define a total range of RF power and positive bias voltages at which MP can develop, these parameters were swept during simulations. Fig.10 shows the barriers expressed in terms of collision current and obtained by scanning input power at different bias voltages. Also Fig.10 shows energy of collision $\mathrm{W}_{\text {_coll }}$ for each barrier.
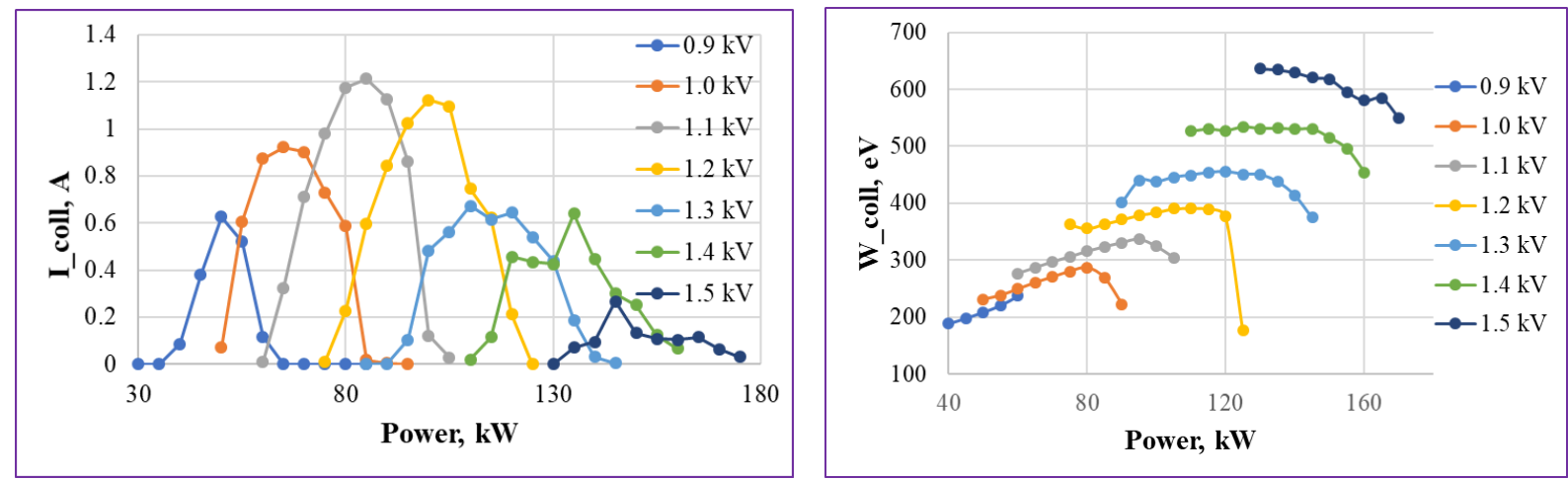

Figure 10: Multipactor barriers in the longer part of the coupler as the functions of RF power and applied high voltage bias. On the right the energy of collision for each barrier vs RF power is shown.

The result confirmed the suggestion that high voltage bias below $\approx 2 \mathrm{kV}$ itself induces multipactor. Fig. 11 shows how positive bias of 0.8-1.5 kV effectively suppresses "normal" MP barriers but induces new ones at the same time.

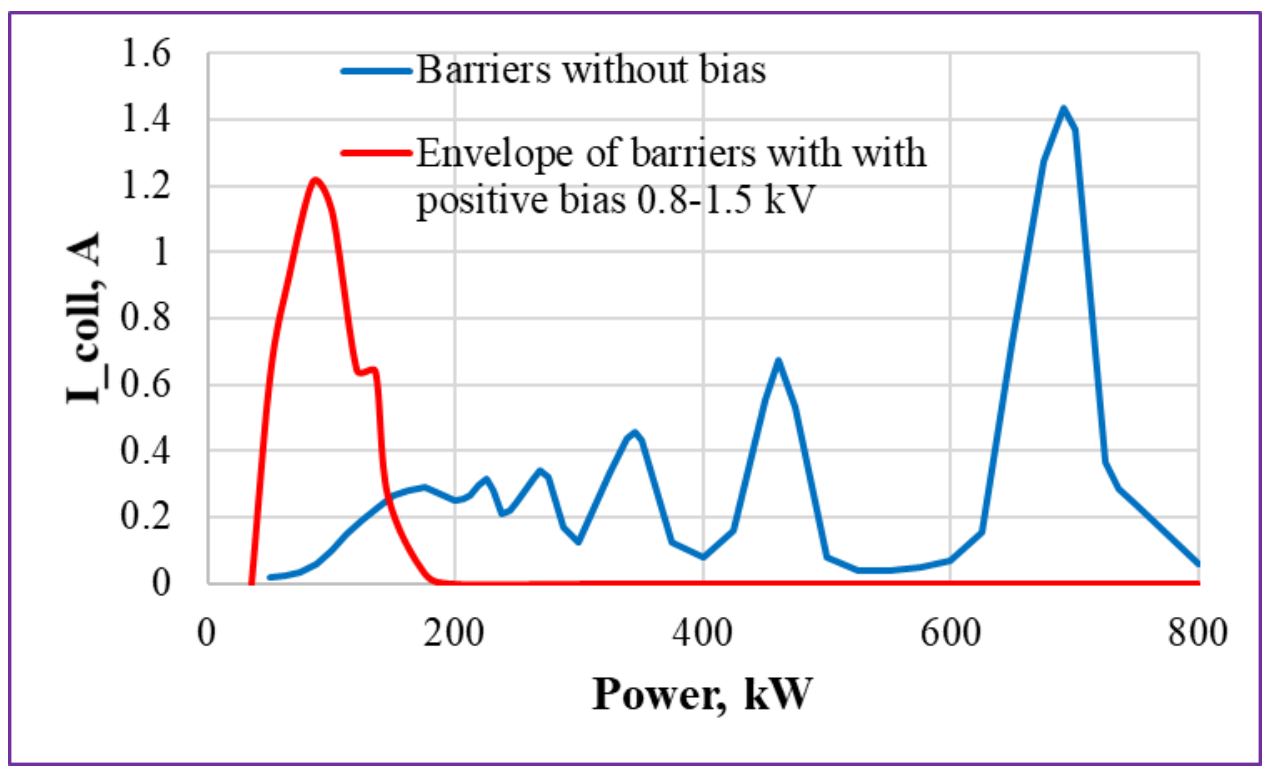

Figure 11: MP barriers in the longer part of the coupler with and without DC bias. 
The coaxial parts of the coupler have relatively narrow gaps between central and outer electrodes, so the electric RF field and electric static field distributions are close to uniform. Besides the maximal distance from the electrodes that particles can fly is about several millimeters from the electrode surfaces. Within such short distances the fields can be considered uniform. For uniform RF field with amplitude $E_{R F}$ and returning uniform electric field $E_{\text {stat }}$ there is an estimation of the MP barrier width [6]:

$$
-\frac{1}{\sqrt{1+\pi^{2}}}<\frac{E_{R F}}{E_{\text {stat }}}<-\frac{1}{\sqrt{4+\pi^{2}}}
$$

This expression was derived for zero initial energy of secondary electrons and defines very narrow area of MP existence. But it indicates that the dynamic conditions for MP development can be fulfilled for rather low RF and static fields with their appropriate ratio. To check this possibility the simulations were repeated with SEY function that has maximum at lower energy of collision to provide emission at lower RF field (see Fig.12). With maximum of SEY and first crossover $\mathrm{W}_{1}$ located at lower $\mathrm{W}_{\text {coll }}$ it was expected to observe MP at lower RF and bias fields, and indeed the simulations confirmed a significant shift of the global MP barrier toward lower RF fields and lower bias voltage (see Fig.13).

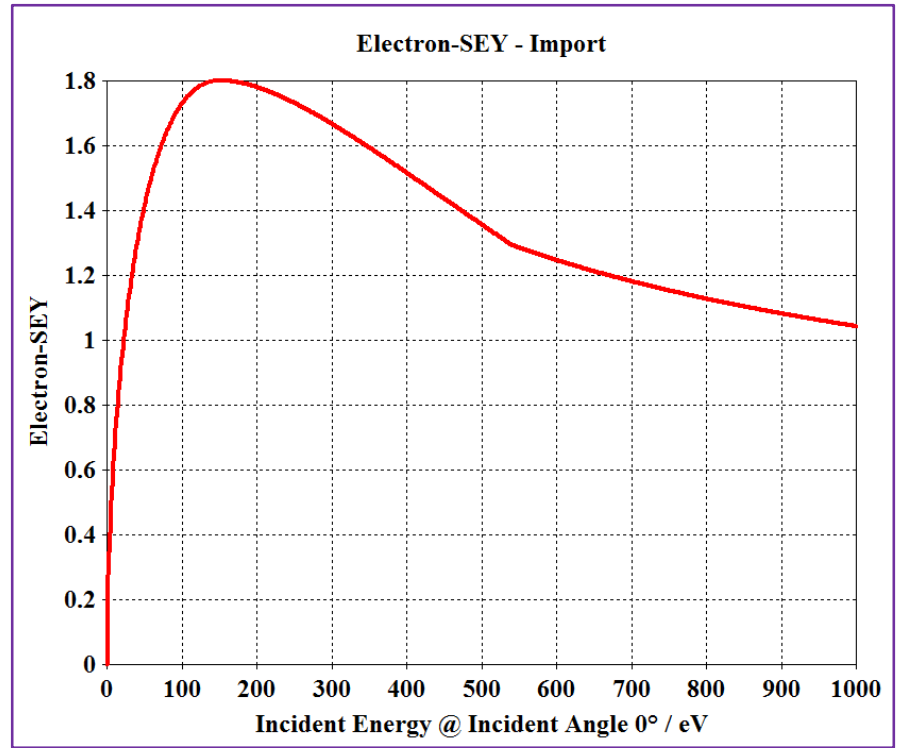

Figure 12: SEY function with maximal yield of 1.8 at $150 \mathrm{eV}$, first crossover $\mathrm{W}_{1}=22.7 \mathrm{eV}$, second crossover $\mathrm{W}_{2}=1125 \mathrm{eV}$. 

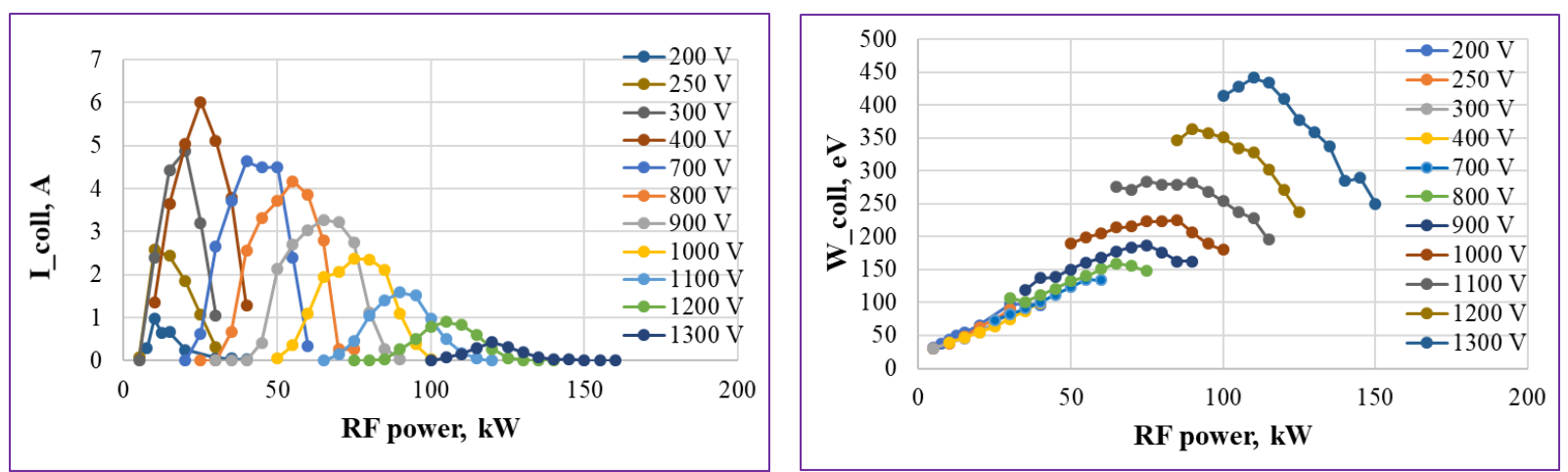

Figure 13: Multipactor barriers in the longer part of the coupler simulated with 1.8@150eV SEY function. On the right $\mathrm{W}_{\text {coll }}$ for each barrier as a function of RF power is shown.

MP barriers widths obtained in the simulations and the simplified analytical estimations are put together in Fig.14.

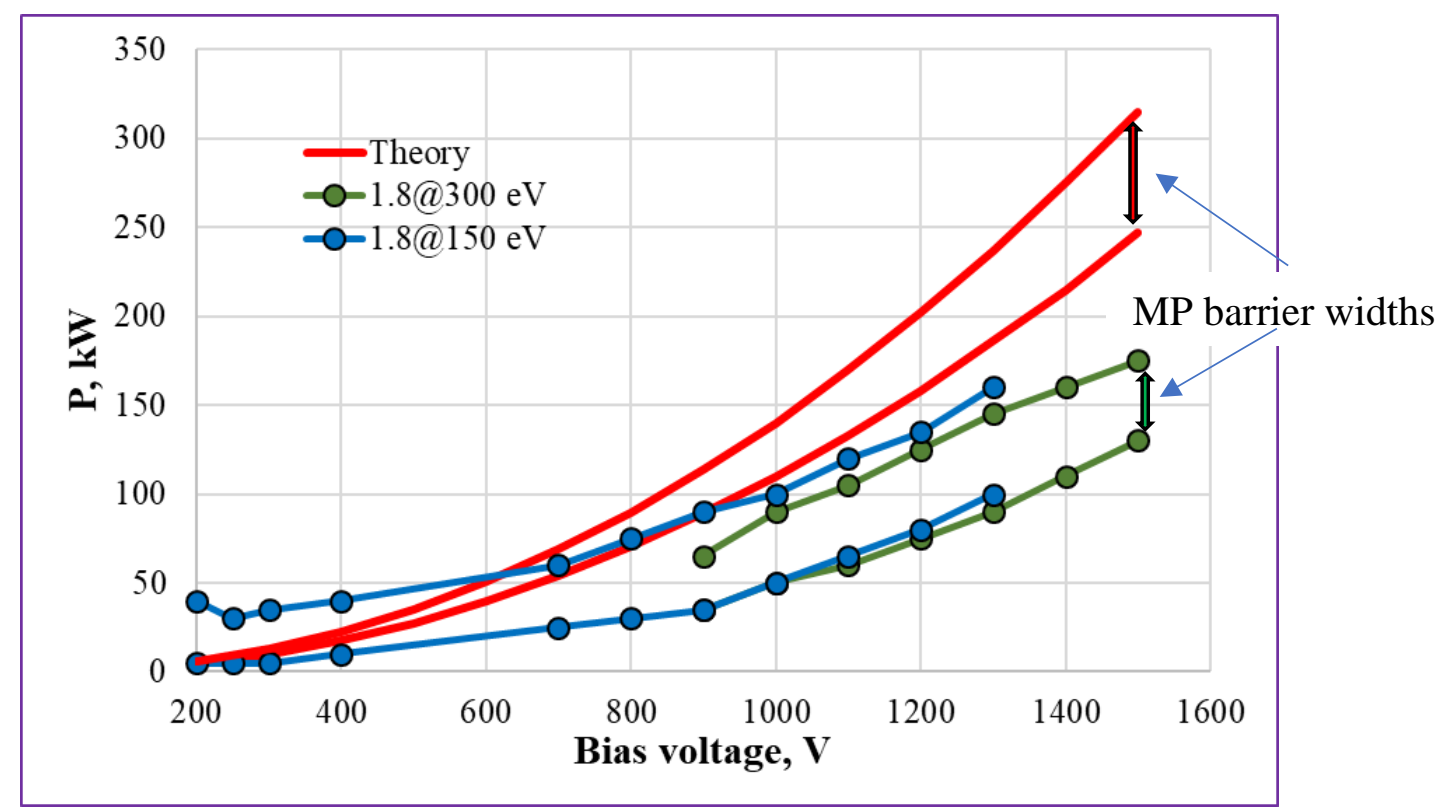

Figure 14: Comparison of the simulated MP barrier widths with simplified theory.

The simulations in the longer part of the coupler were repeated with negative bias. The simulations were performed also with both SEY functions (see Fig.15 and Fig.16) 

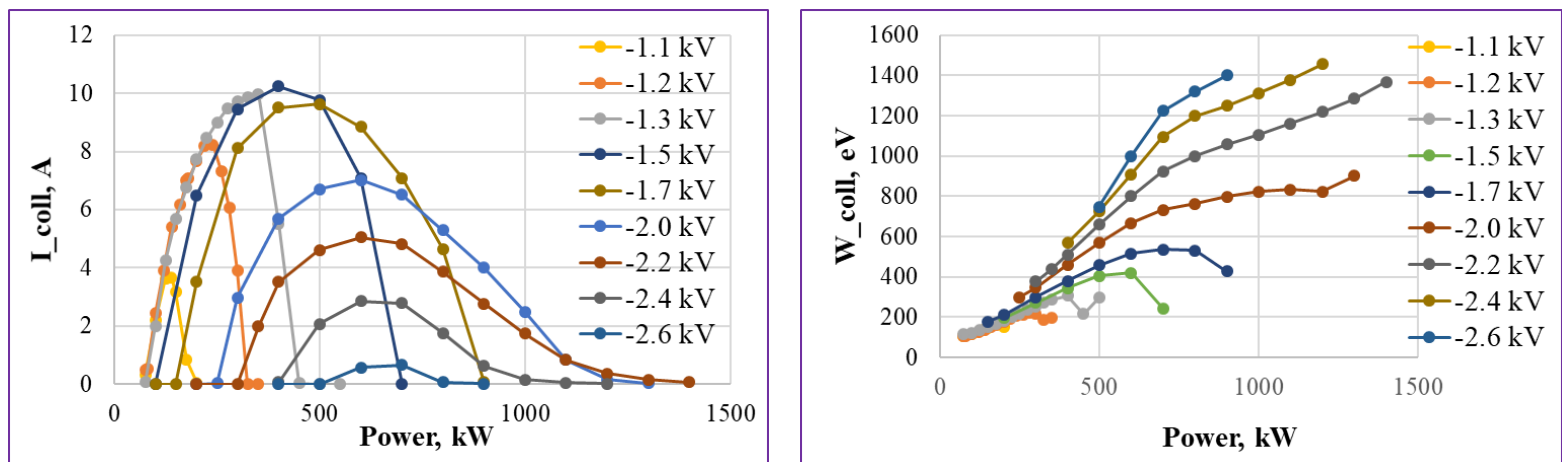

Figure 15: Multipactor barriers in the longer part of the coupler as the functions of RF power and applied negative high voltage bias, SEY function has maximum of 1.8 at $300 \mathrm{eV}$. On the right the energy of collision for each barrier vs RF power is shown.

The intensity of MP with negative bias higher than in previous simulations with positive one. The reason is the larger surface of the outer electrode. MP intensity is limited by current density, so a total collision/emission current is higher for larger emission surface. In general, this part of the coupler probably is a main contributor to MP, because it is much longer than the other parts of the coupler.
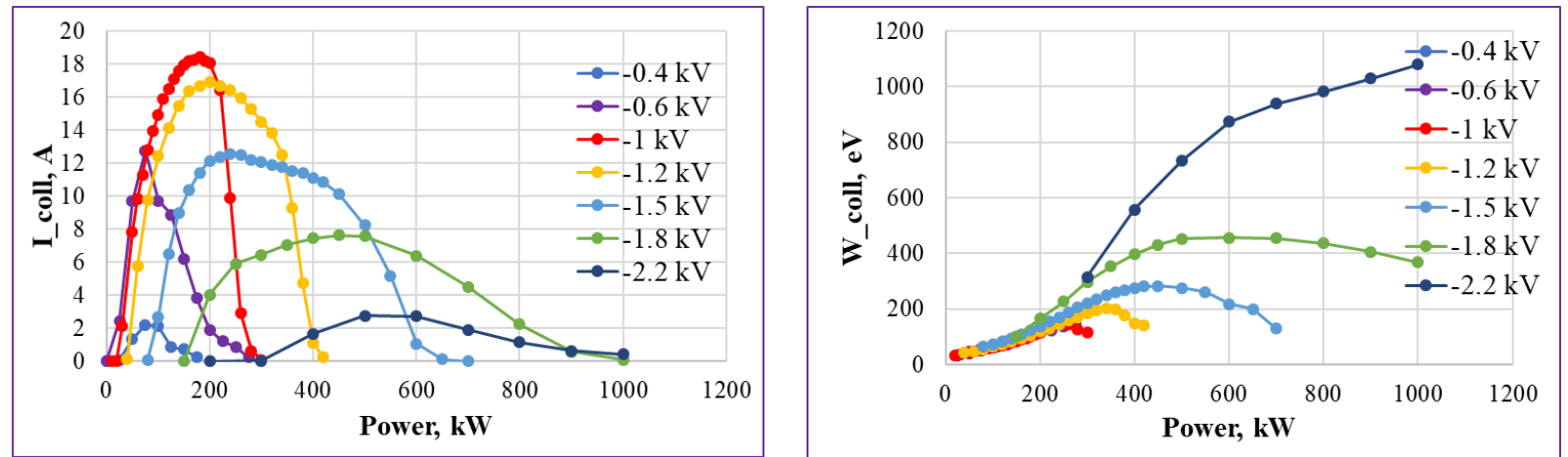

Figure 16: Multipactor barriers in the longer part of the coupler as the functions of RF power and applied negative high voltage bias, SEY function has maximum of 1.8 at $150 \mathrm{eV}$. On the right the energy of collision for each barrier vs RF power is shown.

\section{Multipactor in the shorter part.}

This shorter part of the coupler contains a ceramic window. The high SEY of ceramic and floating potential of the window body due to charging may dominate or significantly change multipactor dynamics. This is not a simple problem, and it requires a special study. In the current study the presence of the window was ignored. The goal of the simulations was just to evaluate MP induced by bias in this particular geometry. The model is a piece of coaxial line with radius of outer conductor $\mathrm{R}=76 \mathrm{~mm}$; radius of inner conductor $\mathrm{r}=33 \mathrm{~mm}$ and length of $150 \mathrm{~mm}$.

For this part of the coupler the same scanning procedure and the SEY function with maximum of 1.8 at $300 \mathrm{eV}$ were used to define MP barriers. The result of the simulations is shown in Fig.17. 

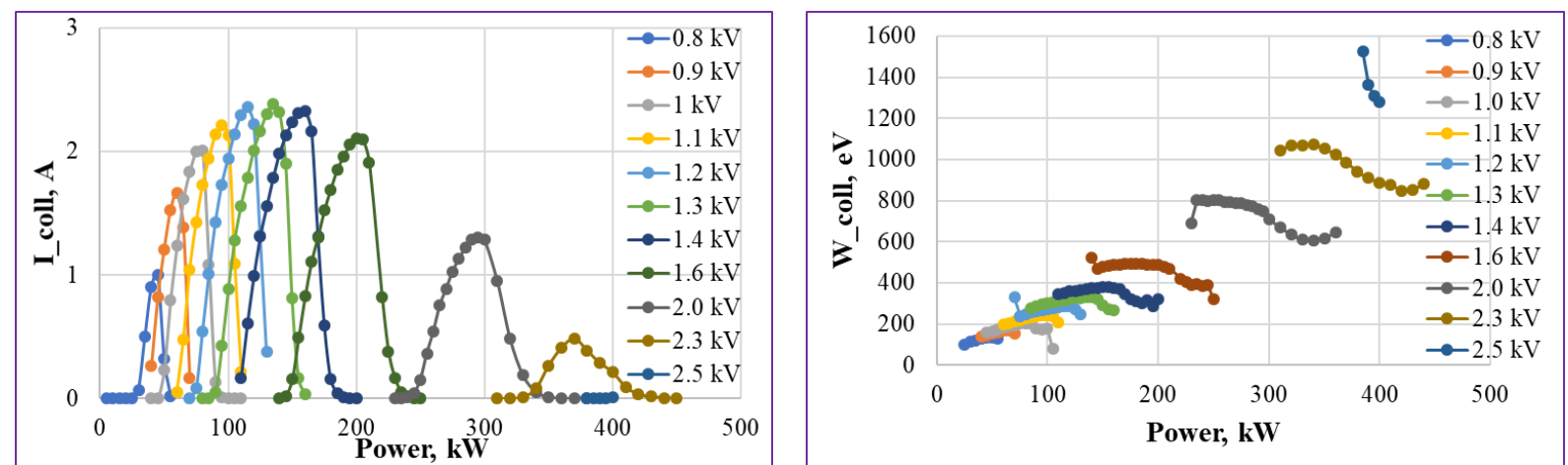

Figure 17: Multipactor barriers in the shorter part of the coupler as the functions of RF power and applied positive high voltage bias. On the right the energy of collision for each barrier vs RF power is shown.

The maximum of MP global barrier in the window part of coupler also confirmed excitation of MP barriers by bias at power levels where they did not exist. The MP interval of power approximately coincides with the one for the longer part, but probably the window part is not a main contributor to the MP process in the coupler because of its short length, though the MP processes on the ceramic surface may change the picture.

\section{Conclusion}

The study confirmed that high voltage bias in the $50 \Omega$ coaxial lines being below $\approx 2 \mathrm{kV}$ induces multipactor barriers. Above $\approx 2 \mathrm{kV}$ all MP barriers including induced ones disappear. Positive bias is preferable since all MP barriers disappear at lower voltage, than with negative one $(1.6 \mathrm{kV}$ vs $2.4 \mathrm{kV}$ ). There is a plan to simulate the lines of different impedances with applied high voltage DC bias to complete the picture.

\section{References}

[1]S.Mitsunobu et al, "High Power Input Coupler for KEKB SC Cavity", Proc. of the 1999 Workshop on RF Superconductivity, Santa Fe, New Mexico, USA.

[2] Y.Kijima et al, "Conditioning of Input Couplers for KEKB Superconducting Cavities”, Proc. of the $10^{\text {th }}$ Workshop on RF Superconductivity, 2001 Tsukuba, Japan.

[3]E.Somersalo et al, "Computational Methods for Analyzing Electron Multipacting in RF Structures", Particle Accelerators, Vol. 59, pp. 107-141, 1998

[4]E.Somersalo et al, “Analysis of Multipacting in Coaxial Lines”, FAE08, PAC95, Dallas, Texas, USA, 1995

[5] https://www.3ds.com/products-services/simulia/products/cst-studio-suite/

[6] И.Н.Сливков, “Процессы при высоком напряжении в вакууме”, Энергоатомиздат, Москва, 1986. 